\title{
Analytical evaluation of extended DRX with additional active cycles for light traffic
}

\author{
Scott Fowler, Ahmed Omar Shahidullah, Mohammed Osman, \\ Johan M. Karlsson and Di Yuan
}

\section{Linköping University Post Print}

\section{Tweet}

N.B.: When citing this work, cite the original article.

Original Publication:

Scott Fowler, Ahmed Omar Shahidullah, Mohammed Osman, Johan M. Karlsson and Di Yuan, Analytical evaluation of extended DRX with additional active cycles for light traffic, 2015, The International Journal of Computer Networks (COMNET), Elsevier, (77), 90-102.

http://dx.doi.org/10.1016/j.comnet.2014.11.020

Copyright: Elsevier http://www.elsevier.com/

Postprint available at: Linköping University Electronic Press http://urn.kb.se/resolve?urn=urn:nbn:se:liu:diva-114286 


\title{
Analytical Evaluation of Extended DRX with Additional Active Cycles for Light Traffic
}

\author{
Scott Fowler ${ }^{\mathrm{a}, *}$, Ahmed Omar Shahidullah ${ }^{\mathrm{a}}$, Mohammed Osman $^{\mathrm{a}}$, Johan Karlsson ${ }^{\mathrm{a}}$, Di Yuan ${ }^{\mathrm{a}}$ \\ ${ }^{a}$ Department of Science and Technology, Linköping University, Norrköping, Sweden, Bredgatan 34, SE-601 74, Norrköping, Sweden
}

\begin{abstract}
LTE and LTE-Advanced mobile technologies have integrated discontinuous reception (DRX) power saving method to optimize the power consumption at the user equipment (UE). The DRX method was proposed by the 3rd Generation partnership Project (3GPP), and since then, the traffic behavior has been analyzed in several studies with a standard 3-state DRX model to describe the trade-off between power saving and delay. In this paper, we presented a novel 4-state and 5-state 3GPP LTE DRX mechanisms. The proposed mechanisms were developed by augmenting (an) active state(s) to deep and/or light sleep cycle of standard 3-state DRX for handling a small burst of packets, thereby bypassing the process of returning to the timer-dependent active mode. We have generated analytical models using a semi-Markov process for bursty packet data traffic and evaluated these augmented DRX mechanisms against a standard 3-state DRX method. Overall, the analytical results from varying timing parameters showed that our augmented DRX (both 4-state and 5-state) improved power saving factor (ranging between 1\% and $8 \%$ ) and reduced delay (ranging between 20\% and 60\%) compared to the standard 3-state DRX. Furthermore, the magnitude of improvement for both delay and power-saving was somewhat greater in 5-state than 4-state.
\end{abstract}

Keywords: LTE, LTE-Advanced, DRX, power saving, delay, semi-Markov process

\section{Introduction}

The latest wireless communication technology being deployed is the fourth generation (4G) mobile technology, known as Long Term Evolution (LTE). LTE had its early specifications in the 3GPP Release 8. The LTE Release 8 uses orthogonal frequency-division multiplexing (OFDM) for downlink multiple access and singlecarrier frequencydivision multiple access (SC-FDMA) for the uplink multiple access, both with a cyclic prefix (CP). As a result, the users benefit from a scalable $20 \mathrm{MHz}$ bandwidth [1]. Further enhancements such as carrier aggregation were added in the LTE Release 10, or otherwise known as LTE-Advanced (LTE-A), thereby enabling bandwidth of $100 \mathrm{MHz}$. Additional features for both LTE and LTE-A, such as advanced antenna techniques (MIMO and SDMA), higher order modulation (64-QAM) and advanced coding systems, have given the privilege of peak data rates to end users devices or user equipment (UE); For downlink, $300 \mathrm{Mbit} / \mathrm{s}$ in LTE and 1Gbits/s in LTE-A, and for uplink, $75 \mathrm{Mbit} / \mathrm{s}$ in LTE and $500 \mathrm{Mbit} / \mathrm{s}$ in LTE-A, respectively[2].

However, there is a huge pitfall for these advancements in mobile technology; the accelerated UE's battery power drainage. Firstly, computational energy requirement became much higher than previous technologies because the latest mobile technologies have made it necessary for receivers to have complex circuitry for computational purposes that drains the UE's battery power quickly. Secondly, energy hungry new services have gained popularity since LTE/LTEA's high data rates and high bandwidth make it possible for new services such as voice, video and multimedia services

\footnotetext{
${ }^{*}$ Corresponding author: Scott Fowler

Email address: scott. fowler@liu.se (Scott Fowler)

${ }^{1}$ Tel: (+46) 11363298

${ }^{2}$ Fax: (+46) 11363270
} 
(e.g. VoIP, video streaming, etc.) to be injected into the network. Consequently, UE's battery power drainage will be even more accelerated. To be able to take full advantage of LTE technologies for utilizing new services, UEs need to efficiently consume the limited power from their battery.

Managing the UE's battery power drainage is an important but difficult issue. One solution is to increase the capacity of the UE's battery, but it has its limitations. Another approach is to efficiently manage the power usage of the UE. The efficient power management can be achieved by turning off the UE during inactive periods and turning on again when there is a need for communication. This mechanism for power saving is known as discontinuous reception (DRX), which was first mentioned in 3GPP Release 7 but proper implementation specifications were provided in 3GPP Release 8.

DRX's power saving performance and its behavior has been studied and described over the past few years in both simulated and analytical works $[2,3,4,5,6,7,8,9,10,11,12,13,14,15,16]$. The first analytical model for the DRX operation was described in the predecessor of the 3GPP LTE-A, Universal Mobile Telecommunications System (UMTS)[17, 8]. The model assumes that packet arrival intervals and transition times follow exponential and general distribution, respectively. Thereafter, several studies $[6,7,9,12]$ have extended the model and developed standard 3 -state DRX models. First, the probabilities that the number of packets arrives depending on the UE's power saving operation during one single packet being served was derived. These models used z-transform to generate a probability function that was differentiated to acquire the average packet transmission delay. The z-transform equations were then converted into Laplace transform equations, and differentiating Laplace transform equations enabled to acquire the durations for the active and sleeping state. Finally, the power saving was obtained by dividing the sleeping state duration by the overall operation time. These standard 3-state DRX models with semi-Markoviian based analytical approach have verified with simulation studies and utilized commonly as a powerful tool to study the trade-off between delay and power saving in DRX.

The trade-off between power saving and delay is one of the main concerns in the development of DRX mechanism. For example, when UMTS DRX (a simple 2-step method of the On and Off durations) and a standard LTE DRX (a 3-state DRX) were compared [12], the LTE DRX resulted in an improved power saving performance. However, this power saving came at the cost of a very large wake-up delay. In a way, the previous studies of standard DRX mechanisms have focused only on one area that is elimination of power consumption wastage during the inactivity periods of an UE. There should be the areas yet be explored for more improvement in power saving without paying the price of large wake-up delays. Therefore in this paper, we will present a new approach by augmenting DRX mechanisms with the single-packet-active state (or stationary active site) embedded in the sleep cycle. (The resulting models will be referred to as 4-state or 5-state model.) This technique improved the DRX's power saving while reducing the wake-up delays compared to the standard DRX method because it is sensitive to the traffic patterns and able to shift between On and Off rapidly. The contribution of the paper are the following: 1) Analysis of the tradeoff of power saving and delay for a small burst of packets while in LTE DRX is in sleep mode, 2) The LTE DRX mechanism has been extended to a 4-state and 5-state DRX to enhance performance (power saving and delay) for a small burst of packets with the DRX sleep cycles, 3) Compared the analytical results of a standard 3-state DRX method against our extended 4-state and 5-state DRX model, 4) Generated analytical models which were modeled with bursty packet data traffic using a semi-Markov process and 5) Our overall contribution is that we demonstrated the addition of states to the generic model, though it is a simple technique, significantly improved the delay and energy efficiency without sacrificing each other.

\section{Background and Related Works}

\subsection{Background}

The 3rd Generation Partner Project (3GPP) initially incorporated DRX during their Release 8 documentations [18]. At that time the widely implemented mobile network was the UMTS network or the $3 \mathrm{G}$ mobile network in which DRX was being put into operation to prolong the battery lifetime. The preliminary specification of DRX implemented on the $3 \mathrm{G}$ mobile networks was a simple 2-step method of the 'ON' and 'OFF' duration. When data are exchanged between the eNodeB and the UE, the DRX will be in 'ON' duration. On the other hand when there is no data to transfer, the DRX will be in the 'OFF' duration and there is an opportunity for some power saving by turning off the RF circuitry of the UE. This 2-step DRX method was later expanded to a 3-step method to allow more power 
saving during inactive periods of the UE, and this 3-step DRX process is implemented in the next generation mobile network known as LTE/LTE-A (henceforth referred to as LTE).

\subsection{Related Work}

To date, several power saving schemes for efficient DRX operation have been proposed and analytically studied, while other studies have just focused on the analysis of the existing 3GPP approved DRX technique.

During the early implementation of DRX in the 3G mobile network, [9] developed analytical and simulation models to study the DRX performance mainly for Poisson traffic. In this study, a novel semi-Markov process to model the 2-step UMTS DRX with bursty packet data traffic was proposed, and the study also investigated the effects of the two DRX parameters on output measures including the power saving factor and the mean packet waiting time.

Later, the DRX mechanism was further enhanced to a 3 stage process. In [2], a detailed power saving method was provided. The study described the effective use of DRX method in both network attached and network idle modes as outlined in LTE, and analytical/simulation results were presented to show the power saving, connection re-establishment and packet delay.

A 3-state LTE DRX was modeled with bursty packet data traffic using semi-Markov process in [6] and [12].The model consists of two types DRX sleep cycles, i.e. DRX short and long sleep cycle. When the UE is in power saving mode (i.e. OFF duration of UE), the next packet delivery will not happen until the UE wakes up (i.e. ON duration of UE). For this reason, DRX short or long cycle introduces delay to packet data that is known as the wake-up delay. Therefore, a trade-off relationship between the wake-up delay and power saving has been shown in these studies. This type of 3-state DRX model with semi-Markov process is now considered to be a standard model and commonly utilized in analytical studies for DRX power saving.

In [19], a Counter-Driven Adaptive DRX (CDA-DRX) scheme has been proposed and proposed and analytically assessed with semi-Markov process. Sine 2-level DRX scheme may incur signaling overhead, the CDR.DRX adjusts the DRX cycle adaptively and autonomously. In this scheme, a series of DRX cycles rather than two DRX cycles are configured and sent to UE via RRC signaling. Two counters are activated to keep tracks of UE activity level in both UE and eNodeB. One counter counts consecutive active DRX periods while the other counts consecutive silent DRX periods. When one of the counters reaches the threshold, the UE and the eNodeB adjust their DRX/DTX cycle (extend or reduce) since they have the same knowledge of data transmission between them. This adjustment of DRX/DTX cycle happens without any RRC or MAC signaling [19].

The authors of [16] presented a LTE DRX model with bursty and Poisson traffic. The main drawback of this model is the assumption that a packet (always) arrives during the sleep period and it has to be delayed and buffered. This assumption ignores the possibility that a packet may arrive during the ON part of a cycle, and allows for the eNodeB right away. Consequently, inaccurate estimates for the power-saving factor and average latency would result.

Other DRX modeling studies focused on specific traffic applications such as HTML [13] and VoIP [14]-[15], and the optimial parameter configuration under diverse arrival rates was explored. The weakness of these studies is that because of the specific assumption on traffic types, the proposed solutions are not generally applicable for various Mobile Internet applications running on an UE.

These ground works for the effective implementation of DRX mechanism are the main inspiration for the current study. Here, we will step forward and shift the focus to the way to handle a small bursty of packets which has never been considered in the previous analysis.

\section{Evaluation of DRX}

\subsection{GPP DRX Mechanism in LTE}

To fully grasp the notion of DRX it is essential to understand the following terms that are related to DRX [18]. These are described below.

- Active state/On state: When a UE is actively monitoring the RF spectrum for data frames.

- Active Time: The time data packets are received by a UE.

- DRX Inactivity Timer: A timer used to initiate the sleep states. 
- Sleep state/Off state: When an UE has powered down and not monitoring the RF spectrum.

- Listen state: A very short period of time when the UE powers up during DRX short cycle to monitor the RF spectrum for incoming Physical Downlink Control Channel (PDCCH) transmissions.

- DRX Short Cycle: A period of time when the UE is in sleep state but periodically transitions to the listen state to monitor the PDCCH for incoming data frames.

- DRX Short Cycle Timer: During the DRX short cycle the DRX Short Cycle Timer sets off the listen state.

- DRX Long Cycle: A time span longer than the DRX Short Cycle where an UE stays in sleep state and wakes up only at the end of the cycle to monitor the PDCCH.

- DRX Long Cycle Timer: During the DRX long cycle the DRX long Cycle Timer set off the listen state.

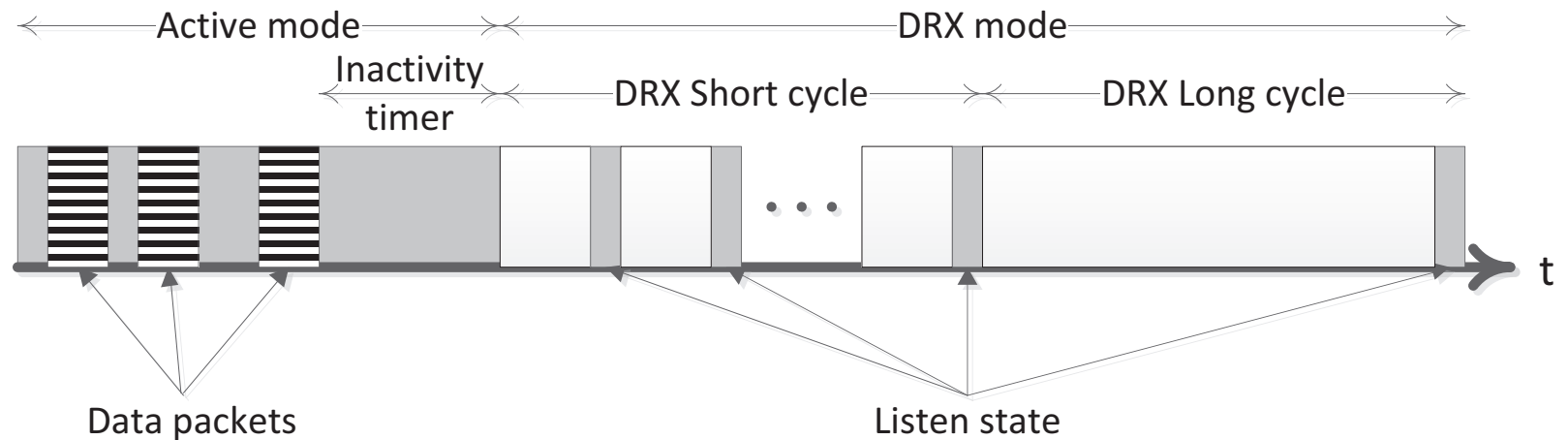

Active state

Sleep state

Figure 1: 3GPP LTE DRX timing diagram

The DRX algorithm as depicted in Figure 1 begins with the DRX inactivity timer counting down from a specified value after a packet has been received by an UE. If a packet arrives before the DRX inactivity timer expires then the timer restarts after receiving the new packet. But if the timer expires and there is no new incoming packet then the UE goes into the light sleep cycle called DRX short cycle. During the DRX short cycle the DRX short cycle timer expires and UE goes into listen state to check for the PDCCH. If the PDCCH indicate new transmission during the listen state then the UE transitions to active state and the DRX algorithm starts over again. However, if the PDCCH indicates no new transmission during the listen states and the DRX short cycle expires then a deep sleep cycle called DRX long cycle is initiated. During the DRX long cycle the UE remains powered down until the DRX long cycle timer expires and then listen state is activated to again check the PDCCH for new incoming packet. Finally, when there is a new packet arrival indication in the listen state after a DRX long cycle timer expires then the UE switches to active state and the process start repeats [18].

In LTE the Radio Resource Control (RRC) manages the DRX operation and thus the DRX can be enabled in either of the RRC modes, RRC_CONNECTED or RRC_IDLE[2]. Being in RRC_IDLE mode there are no packet call sessions, our research will be focused on the DRX performing in the RRC_CONNECTED state where if improperly configured would cause a degraded quality of service (QoS) of the network [6]. During the RRC_CONNECTED mode the following parameters are controlled and configured through RRC [18]:

- DRX inactivity timer $\left(t_{I}\right)$

- DRX short cycle timer $\left(t_{N}\right)$ 
- DRX short cycle $\left(t_{D S}\right)$

- DRX long cycle $\left(t_{D L}\right)$

- Duration of Listen state $(\tau)$

\subsection{Proposed Extensions on 3GPP DRX Mechanism}

The volume of data exchanging between an UE and an eNodeB depends on the type of communication the two entities are having. There can be a large amount of data interchanging between UE and eNodeB for communications like VoIP, video streaming, online gaming etc. On the other hand there can be a small amount of data for communications like UE update messages, synchronization messages etc. In either case for heavy or light data traffic, an incoming packet during any sleep cycle causes the UE to moves to the active state and starts the DRX process from start as described before. However, in the case of light data traffic if there was a way to receive packets and still stay in the sleep cycle then the power saving mechanism will be more efficient. From this idea an extension in the conventional 3GPP DRX algorithm has been proposed by considering additional active states for light traffic burst.

The extension that is being proposed is that during a sleep cycle the UE can transition to active state only to receive a single packet or small burst of packets. After receiving a single packet or small burst of packets, the UE goes back to the sleep cycle from which it was awoken from. This variation to the DRX mechanism will only improve the network's efficiency for light traffic but for heavy traffic the effect will be opposite. Thus a threshold value is needed to distinguish light traffic from heavy traffic and to indicate when the proposed variations or extensions to the DRX will be deactivated.

Case 1:

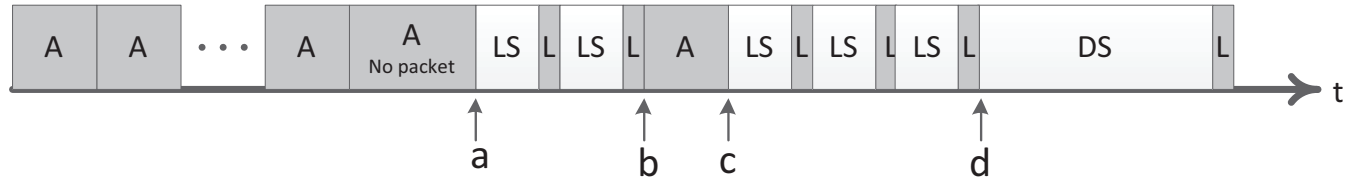

Case 2:

\begin{tabular}{|c|c|c|c|c|c|c|c|c|c|c|c|c|c|}
\hline A & A & $\cdots A$ & $\begin{array}{c}\text { A } \\
\text { No packet }\end{array}$ & LS L & LS & L $\quad A$ & LS & L A & LS & A & LS & $\mathrm{L}$ & A \\
\hline
\end{tabular}

\section{UE is powered on \\ UE is powered off}

$\begin{array}{lll}\text { A } & - & \text { Active State } \\ \text { LS } & - & \text { Light Sleep State } \\ \text { DS } & - & \text { Deep Sleep State } \\ \text { L } & - & \text { Listen State }\end{array}$

Figure 2: Proposed Extensions on the 3GPP DRX timing diagram

Consider setting the threshold value is set to 2 packets, which means that if more than two incoming packets are received then it will be considered as heavy traffic. It means after receiving more than two packets the algorithm of the conventional DRX will be followed. This is illustrated in Figure 2 where two cases are illustrated, one for light traffic and the other for heavy traffic. In case 1 of Figure 2 the DRX light sleep cycle has been initiated at point 'a'. During the light sleep state, the listen state at point ' $b$ ' has an incoming packet notification and a single-packet active state is initiated to receive one packet at point 'c'. In the conventional DRX method the UE would have transitioned to the active state and initiated sleep state after the expiring of the inactivity timer. In case 2 of Figure 2, we have scenario of heavy traffic where during the light sleep state more than two listen states have notification of incoming packets, i.e. point ' $b$ ', point 'd' and point ' $\mathrm{f}$ '. Finally the UE switches to active state and waits for the inactivity timer to expire which is the conventional DRX method. Similar to the light sleep state, the deep sleep cycle will also have the same variation in the transition sequence. In this way, for light traffic, the power of the UE is not wasted to go to sleep mode by waiting for the inactivity timer to expire. 


\section{Performance Analysis of DRX}

\subsection{ETSI Packet Traffic Model}

The ETSI Packet Traffic Model has been adopted by previous studies to represent the network traffic patterns when analyzing the DRX mechanism $[6,9,12]$. Since our study will show the performance analysis of the enhancements made to the conventional 3GPP LTE DRX method, the same traffic model has been applied.

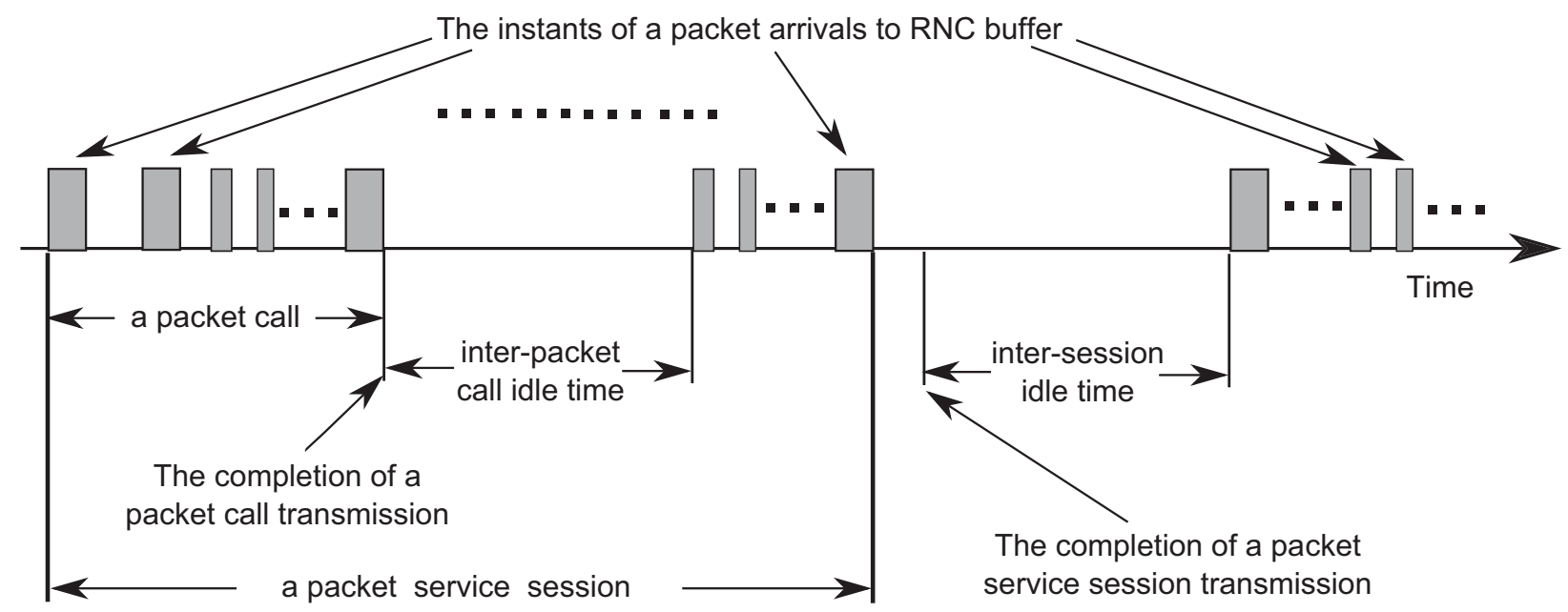

Figure 3: ETSI packet traffic model [9]

Figure 3 depicts the ETSI packet traffic model, where it has been assumed that packet data traffic consists of several packet service sessions. Depending on different applications, each session contains one or more packets call [9]. Several packets might be generated during a packet call which comprises of bursty sequence of packets [20]. The streaming video may consists of one packet call per packet service session while the web browsing comprises sequences of packet calls per packet service session. When the UE initiates a request for information, (for example downloading of a WWW page) a burst of packets will be transmitted to the UE through eNodeB after accepting its request. In this process, a current packet call will be completed while the eNodeB receives positive acknowledgement for the last packet of the packet call from the UE. After the completion of a packet call the eNodeB starts the next new packet call. The time interval between the end of one packet call and the beginning of the next packet call is referred to as inter-packet call idle time, $\left(t_{i p c}\right)$. As a packet service session consists of one or more packet calls, after receiving all packets of these packet calls of the ongoing session, the UE will experience an idle time before beginning of next new session. The time between the end of last session and the beginning of a new session is referred to as inter-session idle time, $t_{i s}[9]$.

For our traffic model, the statistical distributions of the parameters are summarized as follows [9, 12]:

- Inter-session idle time $\left(t_{i s}\right)$ is modeled as an exponential distribution with mean $1 / \lambda_{i s}$.

- Number of packet calls per session $\left(N_{p c}\right)$ follows geometric distribution with mean $\mu_{p c}$.

- Inter-packet call idle time $\left(t_{i p c}\right)$ is assumed to be an exponential distribution with mean $1 / \lambda_{i p c}$.

- Number of packets per packet call $\left(N_{p}\right)$ is modeled as a geometric distribution with mean $\mu_{p}$.

- Inter-packet arrival time $\left(t_{i p}\right)$ is drawn from an exponential distribution with mean $1 / \lambda_{i p}$. 


\subsection{Performance Evaluation Parameter}

The measure of any power saving mechanism is evaluated through the amount of time spent in sleep modes. However, during sleep modes packet reception is not possible and the UE need to wake-up for reception of any incoming packet. This causes a delay which is the time taken by a receiving device to wake-up and receive an incoming packet. Thus we have a trade-off relationship between power saving and the wake-up delay and by comparing this trade-off relationship of different power saving mechanisms we can reveal the strength and weaknesses of different power saving techniques. For the purpose of this study the different DRX mechanisms proposed in the current paper will also be compared through the trade-off relationship of power saving and wake-up delay. These two parameters are defined as:

- Power Saving $(P S)$ : The percentage of time the UE has been kept in sleep mode during both short and long sleep cycle.

- Wake-up Delay $(D)$ : The mean time a packet call transmission is waiting for an UE to wake-up.

These two parameters are discussed in detail in the next section and will help us understand how the proposed extensions to the conventional DRX technique are able to improve the efficiency of the power management of an UE.

\subsection{Analytical Models of the DRX mechnism}

The DRX algorithm is broken down and modeled into 3 states by using the semi-Markov process which has been done in [6] and [12]. Figure 4 displays the 3-state model where it implies that the active state, light sleep state and deep sleep state are represented by state $1\left(S_{1}\right)$, state $2\left(S_{2}\right)$ and state $3\left(S_{3}\right)$, respectively. The modification done to the conventional DRX mechanism was inspired from the work done in [19] where data packets are received during a DRX cycle. Thus the theme of introducing another intermediate active state attached with the sleep states was proposed. By adding active states to the sleep cycles of the conventional DRX mechanism we have extended the 3-state DRX model into a 4-state DRX model and a 5-state DRX model. Firstly, attaching an active state with the light sleep state, a 4-state DRX model is obtained which means that the state $4\left(S_{4}\right)$ is the active state which is connected to $\left(S_{2}\right)$ as shown in Figure 5. Similarly a 5-state DRX model, displayed in Figure 6, can be attained through connecting active states to both the light and deep sleep cycles i.e. state $4\left(S_{4}\right)$ is attached to state $2\left(S_{2}\right)$ and state $5\left(S_{5}\right)$ with the state 3 $\left(S_{3}\right)$. After defining the states of the new proposed DRX techniques, the transitions between the states are also defined through the semi-Markov process to obtain an embedded Markov chain and to derive the state-transition probabilities, $p_{i, j}$ (where $i, j \in\{1,2,3\}$ for 3-state DRX, $i, j \in\{1,2,3,4\}$ for 4-state DRX and $i, j \in\{1,2,3,4,5\}$ for 5 -state DRX).

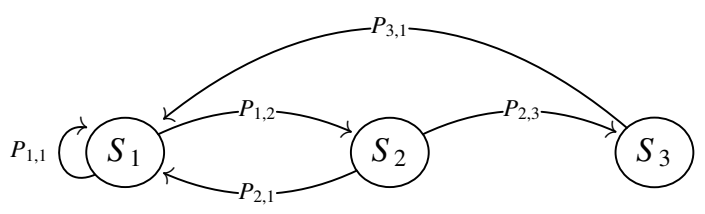

Figure 4: 3-state DRX Model

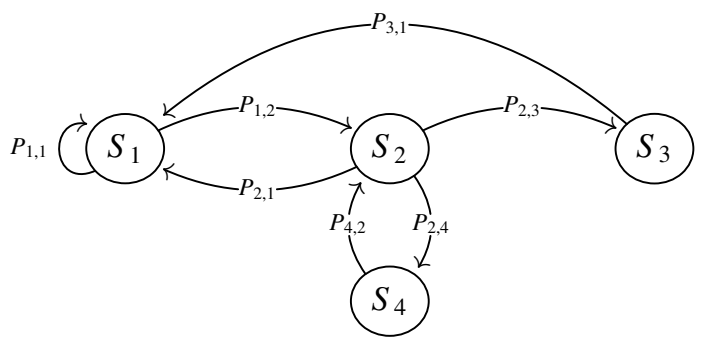

Figure 5: 4-state DRX Model 


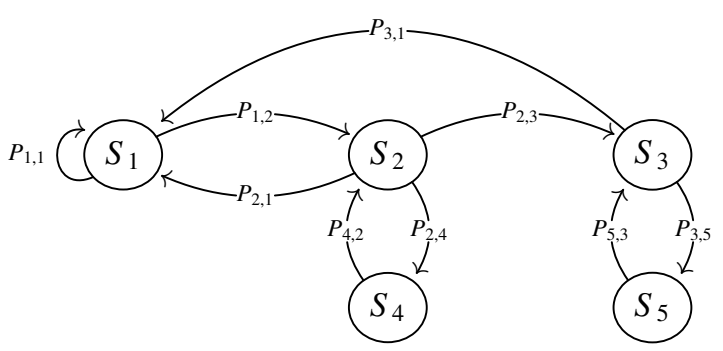

Figure 6: 5-state DRX Model

In relation with the ETSI packet traffic model, depending on the time interval between two consecutive packet calls, a new packet call may start during the ongoing session or a new session will start where the packet call will be the first packet call of that session. The inter-arrival time between two successive packet calls may be the interpacket call idle time $\left(t_{i p c}\right)$ with the probability $P_{p c}=1-1 / \mu_{p c}$ or the inter session idle time $\left(t_{i s}\right)$ with the probability $P_{s}=1 / \mu_{p c}$ [12]. Since there are 3 different DRX analytical models to deal with, we start with the states that are common among all three. Firstly, the Active state or $S_{1}$ where the UE stays in power active mode i.e. the UE monitors the physical channel for the PDCCH and stays awake during the whole packet transmission. In this state, after successfully receiving every packet the UE activates a DRX inactivity timer $\left(t_{I}\right)$. If any packet arrives before the expiration of $t_{I}$ the UE stays in $S_{1}$ and receives all packets delivered from eNodeB. As a result, during an ongoing session, the probability of a new packet call starting before the expiration of $t_{I}$ is $q_{1}=\operatorname{Pr}\left[1 / \lambda_{i p c}<t_{I}\right]=1-e^{-\lambda_{i p c} t_{I}}$ and the probability of a new session starting before the expiration of $t_{I}$ is $q_{2}=\operatorname{Pr}\left[1 / \lambda_{i s}<t_{I}\right]=1-e^{-\lambda_{i s} t_{I}}$. Consequently, if there are no new sessions or packet calls during a session and the $t_{I}$ expires then the UE switches from state $S_{1}$ to state $S_{2}$ as shown in Figures 4, 5 and 6. Ultimately there can be only two transitions that can occur from $S_{1}$ and the probabilities for those transitions can be defined as:

$$
\begin{gathered}
P_{1,1}=P_{p c} q_{1}+P_{s} q_{2} \\
P_{1,2}=P_{p c}\left(1-q_{1}\right)+P_{s}\left(1-q_{2}\right)
\end{gathered}
$$

After exiting the active state the UE moves to the light sleep state which is State $2\left(S_{2}\right)$ in the analytical models. In this state the UE stays in sleep period for a short period of time i.e. UE follows the short DRX cycles. In the beginning of the $\left(S_{2}\right)$ DRX short cycle timer $\left(t_{N}\right)$ is activated. This state consists of sequences of DRX short cycles $\left(t_{D S}\right)$. Every DRX short cycle $\left(t_{D S}\right)$ comprises short 'OFF' duration (sleeping periods, $\left.T_{L S}\right)$ and very small ON periods (listening period, $\tau)$ i.e $\left(t_{D S}=T_{L S}+\tau\right)$. Moreover there are $N_{D S}$ number of DRX short cycle $\left(t_{D S}\right)$ during DRX short cycle timer $\left(t_{N}\right)$ as shown in Figure 1, or in other words $N_{D S}=t_{N} / t_{D S}$. During the DRX short cycle ON duration, or $\tau$ duration, the UE listens to the PDCCH channel for any schedules for packet arrivals and if there is no packet arrival and $\left(t_{N}\right)$ expires the UE switches to deep sleep state $\left(S_{3}\right)$. Alternatively, if there is indication of packet arrival before expiration of $\left(t_{N}\right)$ the UE exits from $S_{2}$ and this is where the standard 3-state DRX differs from the extended 4-state and 5-state DRX method. In a 3-state DRX the UE exits from $S_{2}$ and enters $S_{1}$ but for the extended DRX methods the UE exits from $S_{2}$ and enters the single-packet-active state $\left(S_{4}\right)$. Similar to the active state, the probabilities for a new packet call during a session and for a new session initiation can be also expressed in the light sleep state which are, $q_{3}=\operatorname{Pr}\left[1 / \lambda_{i p c}<t_{N}\right]=1-e^{-\lambda_{i p c} t_{N}}$ and $q_{4}=\operatorname{Pr}\left[1 / \lambda_{i s}<t_{N}\right]=1-e^{-\lambda_{i s} t_{N}}$.

At this point the transition probabilities are expressed but it should be noted that the transition probabilities of extended 4-state DRX and 5-state DRX are different than those of the 3-state DRX model derived in [6] and [12]. In studies [6] and [12] the transition probabilities $P_{2,1}$ and $P_{2,3}$ were derived but we need a third transition probability which is $P_{2,4}$ since there is an extra state attached to state $S_{2}$ as shown in Figure 5. Moreover, as mentioned earlier it is necessary for the extended DRX methods to differentiate between light traffic and heavy traffic by using a threshold value for the number of packets received. Thus we define $T h r_{2,4}$ as the maximum number of packets that can be received during the light sleep cycle before UE goes to $S_{1}$ or in other word the maximum number of times the UE can move to the single-packet-active state or $S_{4}$. Finally, we obtain the transition probabilities of the light sleep state for the extended DRX methods as: 


$$
\begin{gathered}
P_{2,1}=P_{p c} q_{3}^{\left(T h r_{2,4}+1\right)}+P_{s} q_{4}^{\left(T h r_{2,4}+1\right)} \\
P_{2,3}=P_{p c}\left(1-q_{3}\right)+P_{s}\left(1-q_{4}\right) \\
P_{2,4}=1-P_{2,1}-P_{2,3}
\end{gathered}
$$

The next power saving state in the DRX process is the deep sleep state or state $S_{3}$ in the analytical model. In this state the UE goes to sleep for longer period of time $\left(t_{D L}\right)$ i.e. UE follows the long DRX cycles. In case of 3-state and 4-state models there is no single-packet-active state attached to the deep sleep cycle and thus after completing a cycle in $S_{3}$ if a packet is scheduled to arrive an UE moves straight to $S_{1}$. But for the 5-state model if a packet is scheduled to arrive, the UE switches to state $S_{5}$ which is a single-packet-active state. Analogous to the light sleep state, to differentiate between heavy traffic from light traffic we have defined $T h r_{3,5}$ as the maximum number of packets that can be received during the deep sleep cycle before the UE goes to $S_{1}$ or in other word the maximum number of times the UE can move to the single-packet-active state $S_{5}$. This means if packets arrive consecutively and switching from $S_{3}$ to $S_{5}$ reaches a predefined threshold value i.e. $T h r_{3,5}$ then the UE changes its state to $S_{1}$. As like the previous states we define probability for a new packet call during a session in the deep sleep state as $q_{5}=\operatorname{Pr}\left[1 / \lambda_{i p c}<t_{D L}\right]=1-e^{-\lambda_{i p c} t_{D L}}$ and for the probability of a new session initiation we get $q_{6}=\operatorname{Pr}\left[1 / \lambda_{i s}<t_{D L}\right]=1-e^{-\lambda_{i s} t_{D L}}$.

The 3-state DRX and 4-state DRX are similar in the deep sleep state but in the 5-state DRX model a singlepacket-active state is attached to it as shown in Figure 6. As a result, the transition probabilities for the 3-state DRX and 4-state DRX model are identical and can be obtained from the studies [6] and [12]. Continually, just like we derived the transition probabilities for the light sleep state we generated the transition probabilities for the deep sleep state which are:

$$
\begin{gathered}
P_{3,1}=P_{p c} q_{5}^{\left(T h r_{3,5}+1\right)}+P_{s} q_{6}^{\left(T h r_{3,5}+1\right)} \\
P_{3,5}=1-P_{3,1}
\end{gathered}
$$

Lastly, the final states and state transitions that are needed to be explained are the two single-packet-active states and the transition to their respective sleep cycles. For both 4-state and 5-state DRX the single-packet-active state $S_{4}$ is connected to the light sleep state as illustrated in Figures 5 and 6. Since there is only one way for the UE to move i.e. from $S_{4}$ to $S_{2}$ therefore the probability is:

$$
P_{4,2}=1
$$

Equally only in the 5-state DRX the single-packet-active state $S_{5}$ is connected to $S_{3}$ as shown in Figure 6 and the transition for an UE can occur from $S_{5}$ to $S_{3}$ only, therefore we get:

$$
P_{5,3}=1
$$

Putting all the transition probabilities together from Equations (1) to (9) we expressed the probability matrix for all the DRX models. For the 3-state DRX model the transition probability matrix $P_{3-\text { state }}=\left(p_{i, j}\right)$ is taken from [6] and [12]. The transition probability matrices $P_{4-\text { state }}=\left(p_{i, j}\right)$ and $P_{5-\text { state }}=\left(p_{i, j}\right)$ for the 4-state and 5-state DRX are respectively:

$$
\begin{gathered}
\mathbf{P}_{4-\text { state }}=\left(\begin{array}{cccc}
P_{1,1} & P_{1,2} & 0 & 0 \\
P_{2,1} & 0 & P_{2,3} & P_{2,4} \\
1 & 0 & 0 & 0 \\
0 & 1 & 0 & 0
\end{array}\right) \\
\mathbf{P}_{5-\text { state }}=\left(\begin{array}{ccccc}
P_{1,1} & P_{1,2} & 0 & 0 & 0 \\
P_{2,1} & 0 & P_{2,3} & P_{2,4} & 0 \\
P_{3,1} & 0 & 0 & 0 & P_{3,5} \\
0 & 1 & 0 & 0 & 0 \\
0 & 0 & 1 & 0 & 0
\end{array}\right)
\end{gathered}
$$


Let $\pi_{i}(i \epsilon\{1,2,3,4\})$ denote the probability of staying at state $S_{i}(i \epsilon\{1,2,3,4\})$ of the embedded Markov chain in steady state for the 4 -state DRX model. By using equation $\sum_{i=1}^{4} \pi_{i}=1$ and balance equation $\pi_{i}=\sum_{j=1}^{4} \pi_{j} P_{j, i}$ we solved the stationary distribution as displayed in the appendix. This gives us:

$$
\prod=\left\{\begin{array}{l}
\pi_{1}=\frac{1-p_{24}}{1-p_{24}+p_{12}+p_{12} p_{24}+p_{12} p_{23}} \\
\pi_{2}=\frac{p_{12}}{1-p_{24}+p_{12} p_{12} p_{12} p_{24}+p_{12} p_{23}} \\
\pi_{3}=\frac{p_{22} p_{23}}{1-p_{24}+p_{12}+p_{12} p_{24}+p_{12} p_{23}} \\
\pi_{4}=\frac{p_{12} p_{24}}{1-p_{24}+p_{12}+p_{12} p_{24}+p_{12} p_{23}}
\end{array}\right.
$$

In the same way for the 5-state DRX model let $\pi_{i}(i \epsilon\{1,2,3,4,5\})$ denote the probability of staying at state $S_{i}(i \epsilon\{1,2,3,4,5\})$ of the embedded Markov chain in steady state. By solving (also shown in the appendix) the equation $\sum_{i=1}^{5} \pi_{i}=1$ and balance equation $\pi_{i}=\sum_{j=1}^{5} \pi_{j} P_{j, i}$ we get:

$$
\prod=\left\{\begin{array}{l}
\pi_{1}=\frac{\left(1-p_{35}\right)\left(1-p_{24}\right)}{\left(1-p_{35}\right)\left(1-p_{24}\right)+p_{12}\left(1+p_{24} 4 p_{23}+p_{23} p_{35}-p_{35}-p_{24} p_{35}\right)} \\
\pi_{2}=\frac{p_{12}\left(1-p_{35}\right)}{\left(1-p_{35}\right)\left(1-p_{24}\right)+p_{12}\left(1+p_{24}+p_{23}+p_{23} p_{35}-p_{35}-p_{24} p_{35}\right)} \\
\pi_{3}=\frac{p_{21} p_{23}}{\left(1-p_{35}\right)\left(1-p_{24}\right)+p_{12}\left(1+p_{24}+p_{23}+p_{23} p_{35}-p_{35}-p_{24} p_{35}\right)} \\
\pi_{4}=\frac{p_{12} p_{24}\left(1-p_{35}\right)}{\left(1-p_{35}\right)\left(1-p_{24}\right)+p_{12}\left(1+p_{24} 4 p_{23}+p_{23} p_{35}-p_{35}-p_{24} p_{35}\right)} \\
\pi_{5}=\frac{p_{12} p_{23} p_{35}}{\left(1-p_{35}\right)\left(1-p_{24}\right)+p_{12}\left(1+p_{24}+p_{23}+p_{23} p_{35}-p_{35}-p_{24} p_{35}\right)}
\end{array}\right.
$$

The analysis of power saving (one of the performance evaluating parameter) involves the calculation of the time spent in the sleep modes and thus we proceed first in finding the time spent in all the available states. It is assumed that holding time $H_{i}(i \epsilon\{1,2,3,4\})$ for 4 -state DRX and $(i \epsilon\{1,2,3,4,5\})$ for 5-state DRX denote how long UE will stay in a state $S_{i}(i \epsilon\{1,2,3,4\})$ for 4-state DRX and $(i \epsilon\{1,2,3,4,5\})$ for 5-state DRX of semi-Markov process.

The holding time $E\left[H_{1}\right]$ indicates the holding time for $S_{1}$ and since all the DRX models have the identical $S_{1}$ and transitions from $S_{1}$, the final outcome will be considered same for all DRX models. How long UE will stay in state $S_{1}$ depends on sum of the service time for $N_{P}-1$ packet and inactivity period $t_{\text {inact }}^{*}$ for current packet call $[6,12]$. The offered packet-level traffic is, $A=E\left[t_{s}\right] \lambda_{i p}$, where $t_{s}$ denotes the packet service time [6]. Therefore:

$$
E\left[H_{1}\right]=\frac{\mu_{P}-1}{\lambda_{i p}}+E\left[t_{\text {inact }}^{*}\right]
$$

As we know state $S_{1}$ consists of sequences of inactivity periods, if during inactivity period $\left(t_{\text {inact }}^{*}\right)$ the PDCCH indicates the UE there is packet delivery before expiration of $t_{I}$, then the inactivity period should be equal to interpacket call idle time $\left(t_{i p c}\right)$, i.e $t_{\text {inact }}^{*}=t_{i p c}$; If $t_{I}$ expires before the PDCCH indication of packet delivery then the inactivity period equals $t_{I}$, i.e. $t_{\text {inact }}^{*}=t_{I}$. For staying in state $S_{1}$, inter-packet call idle time $\left(t_{i p c}\right)$ or DRX inactivity timer $\left(t_{I}\right)$ should be minimum. Therefore, for Case 1: we have $t_{\text {inact }}^{*}=\min \left(t_{i p c}, t_{I}\right)$ and for Case $2: t_{\text {inact }}^{*}=\min \left(t_{\text {is }}, t_{I}\right)$. Now the equation $E\left[t_{\text {inact }}^{*}\right]$ :

$$
E\left[t_{\text {inact }}^{*}\right]=P_{p c} E\left[\min \left(t_{i p c}, t_{I}\right)\right]+P_{s} E\left[\min \left(t_{i s}, t_{I}\right)\right]
$$

again for Case 1, we have:

$$
E\left[\min \left(t_{i p c}, t_{I}\right)\right]=\frac{1}{\lambda_{i p c}}\left[1-e^{-\lambda_{i p c} t_{I}}\right]
$$

and similarly for Case 2 we have:

$$
E\left[\min \left(t_{i s}, t_{I}\right)\right]=\frac{1}{\lambda_{i s}}\left[1-e^{-\lambda_{i s} t_{I}}\right]
$$

giving us:

$$
E\left[t_{\text {inact }}^{*}\right]=\frac{P_{p c}}{\lambda_{i p c}}\left[1-e^{-\lambda_{i p c} t_{I}}\right]+\frac{P_{s}}{\lambda_{i s}}\left[1-e^{-\lambda_{i s} t_{I}}\right]
$$

The holding time $E\left[H_{1}\right]$ is thus: 


$$
E\left[H_{1}\right]=\frac{\mu_{P}-1}{\lambda_{i p}}+\frac{P_{p c}}{\lambda_{i p c}}\left[1-e^{-\lambda_{i p c} t_{I}}\right]+\frac{P_{s}}{\lambda_{i s}}\left[1-e^{-\lambda_{i s} t_{I}}\right]
$$

As mentioned earlier, state $S_{2}$ is considered as light sleep period of the UE. The light sleep period comprises of a sequences of DRX short cycle $\left(t_{D S}\right)$ where $N_{D S}$ is the number of DRX short cycles. As staying in state $S_{2}$ depends on the DRX short cycle timer $\left(t_{N}\right)$ expiration, we have $N_{D S}=t_{N} / t_{D S}$. In the case of 3-state DRX the holding time for state $S_{2}$ is taken from [6] and [12]. However in state $S_{2}$ for the 4-state and 5-state DRX we also have to consider the extra DRX short cycle due to the transitions to the single-packet-active state. Therefore, for both light and heavy traffic, the first few packet calls or new sessions (depending on the $T h r_{2,4}$ ) will add extra $t_{D S}$ cycles to the $N_{D S}$. Since new packet call or new session may start before or after $t_{N}$ expires, the light sleep period lasts either $N_{D S}^{* *}\left(N_{D S}^{* *}<N_{D S}\right)$, $N_{D S}^{*}\left(N_{D S}^{*}<N_{D S}\right)$ or $\left(N_{D S}+T h r_{2,4}\right)$ DRX short cycles with probabilities $P_{2,1}, P_{2,4}$ or $P_{2,3}$ respectively. Therefore, we have mean holding time in state $S_{2}$ as:

$$
E\left[H_{2}\right]=\left\{P_{2,3}\left(N_{D S}+T h r_{2,4}\right)+P_{2,1} E\left[N_{D S}^{* *}\right]+P_{2,4} E\left[N_{D S}^{*}\right]\right\} t_{D S}
$$

Due to the memoryless property of the exponential distribution of inter-packet call idle time $\left(t_{i p c}\right)$ and inter-session call idle time $\left(t_{i s}\right)$, we can assume that $N_{D S}^{*}$ and $N_{D S}^{* *}$ have a geometric distribution with mean $1 / p_{D S}$, where $p_{D S}$ is the probability that the packet call delivery may happen during a DRX short cycle [12]. Hence:

$$
E\left[N_{D S}^{*}\right]=\frac{P_{p c}}{1-e^{-\lambda_{i p c} t_{D S}}}+\frac{P_{s}}{1-e^{-\lambda_{i s} t_{D S}}}
$$

and similarly,

$$
\begin{aligned}
& E\left[N_{D S}^{* *}\right]=\frac{P_{p c}}{1-e^{-\lambda_{i p c} t_{N}\left(\frac{T h r_{2,4}+1}{T h r_{2,4}+N_{D S}}\right)}}+\frac{P_{s}}{1-e^{-\lambda_{i s} t_{N}\left(\frac{T h r_{2,4}+1}{T h r_{2,4}+N_{D S}}\right)}} \\
& p_{i(4-s t a t e)}=\left\{\begin{array}{c}
\underbrace{P_{p c} e^{-\lambda_{i p c}\left\{t_{I}+[i-1+(m-1)] t_{D S}\right\}}\left(1-e^{-\lambda_{i p c} t_{D S}}\right)+P_{s} e^{-\lambda_{i s}\left\{t_{I}+[i-1+(m-1)] t_{D S}\right\}}\left(1-e^{-\lambda_{i s} t_{D S}}\right)}_{m=\sum_{j=1}^{T h r_{2,4}} k[k=1,2,3, \ldots], \quad 1 \leq i<N_{D S}} \\
\underbrace{P_{p c} e^{-\lambda_{i p c}\left[t_{I}+t_{N}+\left(i-N_{D S}-1\right) t_{D L}\right]}\left(1-e^{-\lambda_{i p c} t_{D L}}\right)+P_{S} e^{-\lambda_{i s}\left[t_{I}+t_{N}+\left(i-N_{D S}-1\right) t_{D L}\right]}\left(1-e^{-\lambda_{i s} t_{D L}}\right)}_{i \geq N_{D S}}
\end{array}\right.
\end{aligned}
$$

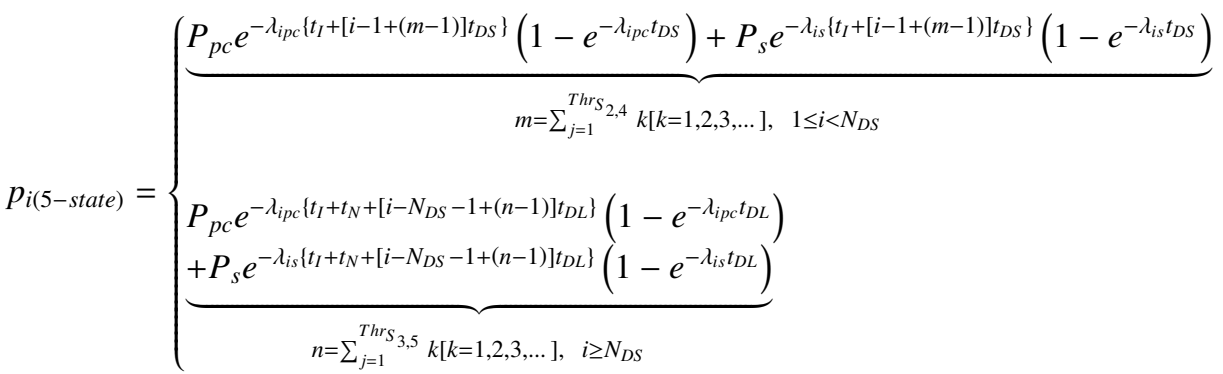

Finally, the holding time of state $S_{2}$ is: 


$$
\begin{aligned}
E\left[H_{2}\right] & =P_{2,3}\left(N_{D S}+T h r_{2,4}\right) t_{D S}+P_{2,4}\left[\frac{P_{p c}}{1-e^{-\lambda_{i p c} t_{D S}}}+\frac{P_{s}}{1-e^{-\lambda_{i s} t_{D S}}}\right] t_{D S} \\
& +P_{2,1}\left[\frac{P_{p c}}{1-e^{-\lambda_{i p c} t_{N}\left(\frac{T h r_{2,4}+1}{T h r_{2,4}+N_{D S}}\right)}}+\frac{P_{S}}{1-e^{-\lambda_{i s} t_{N}\left(\frac{T h r_{2,4}+1}{T h r_{2,4}+N_{D S}}\right)}}\right] t_{D S}
\end{aligned}
$$

During the DRX short cycle period the UE wakes up for certain amount of time to monitor PDCCH transmission. Due to this reason DRX short cycle contains certain ON duration which is denoted as $\tau$. Therefore, the effective sleep time for the DRX short cycle should be $t_{D S}-\tau$; So, the Equation (25) becomes:

$$
\begin{aligned}
E\left[H_{2}^{e f f}\right] & =P_{2,3}\left(N_{D S}+T h r_{2,4}\right)\left(t_{D S}-\tau\right)+P_{2,4}\left[\frac{P_{p c}}{1-e^{-\lambda_{i p c} t_{D S}}}+\frac{P_{s}}{1-e^{-\lambda_{i s} t_{D S}}}\right]\left(t_{D S}-\tau\right) \\
& +P_{2,1}\left[\frac{P_{p c}}{1-e^{-\lambda_{i p c} t_{N}\left(\frac{T h r_{2,4}+1}{T h r_{2,4}+N_{D S}}\right)}}+\frac{P_{S}}{1-e^{-\lambda_{i s} t_{N}\left(\frac{T h r_{2,4}+1}{T h r_{2,4}+N_{D S}}\right)}}\right]\left(t_{D S}-\tau\right)
\end{aligned}
$$

State $S_{3}$ is deep sleep period of the UE where state $S_{3}$ consists of a number of deep sleep periods that comprise $N_{D L}$ DRX long cycles for the 3-state and 4-state DRX cycle. Thus the holding time for state $S_{3}$ can be obtained from [6] and [12]. But similar to light sleep state, for the 5-state DRX the deep sleep cycle lasts either $N_{D L}^{*}$ or $N_{D L}$ DRX long cycles with probabilities $P_{3,5}$ or $P_{3,1}$ respectively. Therefore we can obtain the holding time for $S_{3}$ as:

$$
E\left[H_{3}\right]=\left\{P_{3,5} E\left[N_{D L}\right]+P_{3,1} E\left[N_{D L}^{*}\right]\right\} t_{D L}
$$

Again, similar to the light sleep state for the memoryless property of the exponential distribution of inter-packet call idle time $\left(t_{i p c}\right)$ and inter-session call idle time $\left(t_{i s}\right)$, it can be assumed that $N_{D L}$ has a geometric distribution with mean $1 / p_{D L}$, where $p_{D L}$ is the probability that the packet call delivery may happen during a DRX long cycle [12]. Hence:

$$
E\left[N_{D L}\right]=\frac{P_{p c}}{1-e^{-\lambda_{i p c} t_{D L}}}+\frac{P_{s}}{1-e^{-\lambda_{i s} t_{D L}}}
$$

and similarly,

$$
E\left[N_{D L}^{*}\right]=\frac{P_{p c}}{1-e^{-\lambda_{i p c} t_{D L}\left(\frac{T h r_{3,5}}{T h r_{3,5}+1}\right)}}+\frac{P_{s}}{1-e^{-\lambda_{i s} t_{D L}\left(\frac{T h r_{3,5}}{T h r_{3,5}+1}\right)}}
$$

Finally by substituting Equations (28) and (29) into (27), we can obtain the holding time of state $S_{3}$ :

$$
\begin{aligned}
E\left[H_{3}\right] & =P_{3,5}\left[\frac{P_{p c}}{1-e^{-\lambda_{i p c} t_{D L}}}+\frac{P_{s}}{1-e^{-\lambda_{i s} t_{D L}}}\right] t_{D L} \\
& +P_{3,1}\left[\frac{P_{p c}}{1-e^{-\lambda_{i p c} t_{D L}\left(\frac{T h r_{3,5}}{T h r_{3,5}+1}\right)}}+\frac{P_{s}}{1-e^{-\lambda_{i s} t_{D L}\left(\frac{T h r_{3,5}}{T h r_{3,5}+1}\right)}}\right] t_{D L}
\end{aligned}
$$

As the effective sleep time for DRX long cycle should be $t_{D L}-\tau$; So, Equation (30) becomes:

$$
\begin{aligned}
E\left[H_{3}^{e f f}\right] & =P_{3,5}\left[\frac{P_{p c}}{1-e^{-\lambda_{i p c} t_{D L}}}+\frac{P_{s}}{1-e^{-\lambda_{i s} t_{D L}}}\right]\left(t_{D L}-\tau\right) \\
& +P_{3,1}\left[\frac{P_{p c}}{1-e^{-\lambda_{i p c} t_{D L}\left(\frac{T h r_{3,5}}{T h r_{3,5}+1}\right)}}+\frac{P_{S}}{1-e^{-\lambda_{i s} t_{D L}\left(\frac{T h r_{3,5}}{T h r_{3,5}}\right)}}\right]\left(t_{D L}-\tau\right)
\end{aligned}
$$


Power Saving Factor (PS): Power saving factor determines how much power can be saved in LTE by using DRX mechanism, i.e. the percentage of time the UE has been kept in sleep mode. Power saving factor for the 4-state DRX model can be derived as:

$$
P S_{4-\text { state }}=\frac{\pi_{2} E\left[H_{2}^{\text {eff }}\right]+\pi_{3} E\left[H_{3}^{\text {eff }}\right]}{\sum_{i=1}^{4} \pi_{i} E\left[H_{i}\right]}
$$

Similarly, power saving factor for the 5-state DRX model can be derived as:

$$
P S_{5-\text { state }}=\frac{\pi_{2} E\left[H_{2}^{e f f}\right]+\pi_{3} E\left[H_{3}^{e f f}\right]}{\sum_{i=1}^{5} \pi_{i} E\left[H_{i}\right]}
$$

Wake-up Delay $(\boldsymbol{E}[\boldsymbol{D}])$ : During the DRX short or long cycle the UE does not receive any packet. That is why the eNodeB can not immediately transmit packet to the UE even though the eNodeB has packet in its buffer. The eNodeB has to wait until the UE listens to PDCCH during DRX short or long cycles. The UE monitors the PDCCH in listening interval of DRX short or long cycle. For this reason data packet experiences some delay that is defined as wake-up delay. During the light or deep sleep period a packet call may happen. The probability of starting a packet call during $i^{\text {th }}$ DRX cycle for a 3-state DRX is taken from [6] and [12] and developed for the 4-state and 5-state DRX which are equations (23) and (24) respectively.

Finally, the delay equation can be derived as:

$$
E[D]=\sum_{i=1}^{N_{D S}} p_{i} \frac{t_{D S}}{2}+\sum_{i=N_{D S}+1}^{\infty} p_{i} \frac{t_{D L}}{2}
$$

\section{Numerical Results}

In this study, the performance of the UE power saving and wake-up delay in the novel LTE DRX 4- and 5-state mechanisms were evaluated by the analytical computation. The parameters are set based on the setup of the validated and standardized 3-state DRX model in [6] and [12].

We have used the same ETSI bursty data traffic model for modeling the data traffic for all our DRX models, and the following numerical values were used; $\lambda_{i p}=10, \lambda_{i s}=\frac{1}{2000}, \lambda_{i p c}=\frac{1}{30}, \lambda_{p}=10, \mu_{p}=25$ and $\mu_{p c}=5$. The derivation of power saving and delay shows that the two performance factors (power saving and delay) depend heavily on the timing values $t_{I}, t_{N}, t_{D S}, t_{D L}$ and $\tau$. While changing one timing value we need to keep the other timing values constant. Thus the initial values for the timing values are $t_{I}=2 \mathrm{sec}, t_{N}=10 \mathrm{sec}, t_{D S}=2 \mathrm{sec}, t_{D L}=10 \mathrm{sec}$ and $\tau=0.1 \mathrm{sec}$. The analysis of the timing parameters are broken down into two categories, the timers $\left(t_{I}\right.$ and $\left.t_{N}\right)$ and the cycle times $\left(t_{D S}\right.$ and $\left.t_{D L}\right)$. In the case of the timers, it is established that when $t_{I}$ or $t_{N}$ increases both the power saving factor and the wake-up delay decreases. This indicates the trade-off relationship between the two parameters. The same principle holds for the 3-state as well as augmented DRX mechanisms (4-state and 5-state DRX).

Overall, the augmented DRX mechanisms show an improvement in both the power saving factor and wake-up delay compared to the standard 3-state DRX. The first timing parameter illustrated in Figure 7 is the inactivity timer $\left(t_{I}\right)$. It shows that the 4-state DRX is more effective for improving power saving performance than reduction of the delay. Our analysis shows that he power saving factor has an average increase of approximately $5 \%$ while the delay has a minor average decrease of about $0.5 \%$. The 5-state DRX method not only has better performance than the 3-state DRX but also outshines the 4-state DRX method as shown in Figure 7. In the 5-state, the average increase of power saving factor is approximately $8 \%$ and the average decrease in wake-up delay around $21 \%$ compared to 3 -state. The improvement in power saving of the augmented DRX comes from the following reasons. In case of light traffic, even if packets are incoming the UE does not have to go to active state and stays in the sleep cycles unlike the standard 3-state model. Therefore, even if the $t_{I}$ is increased, the power saving will not decrease in the 4-state and 5-state DRX. Similarly, delay is decreased during light traffic because the UE is not transitioning to the active state and thus there are no delays since the UE does not have to wait for the $t_{I}$ to expire. 


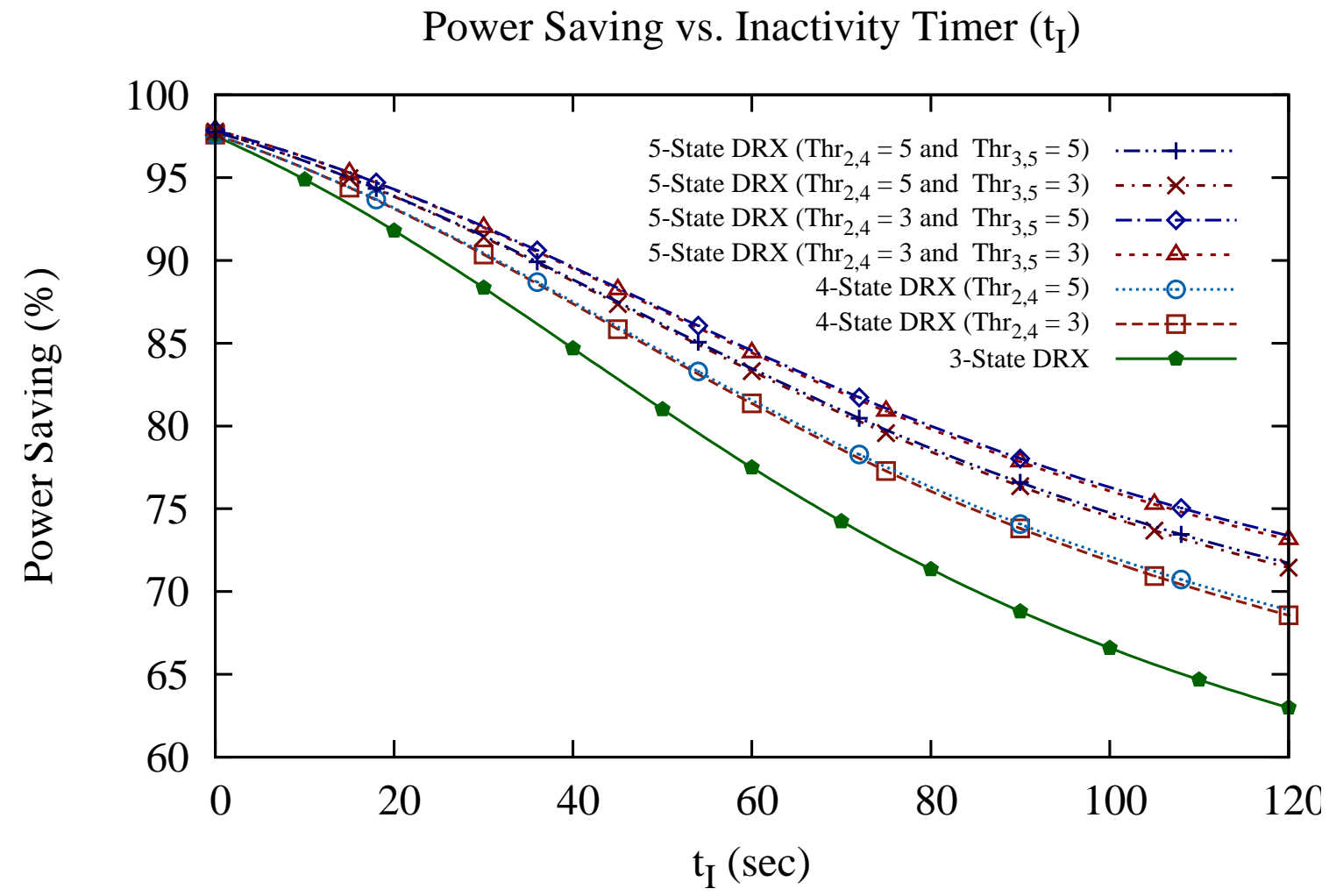

Delay vs. Inactivity Timer $\left(\mathrm{t}_{\mathrm{I}}\right)$

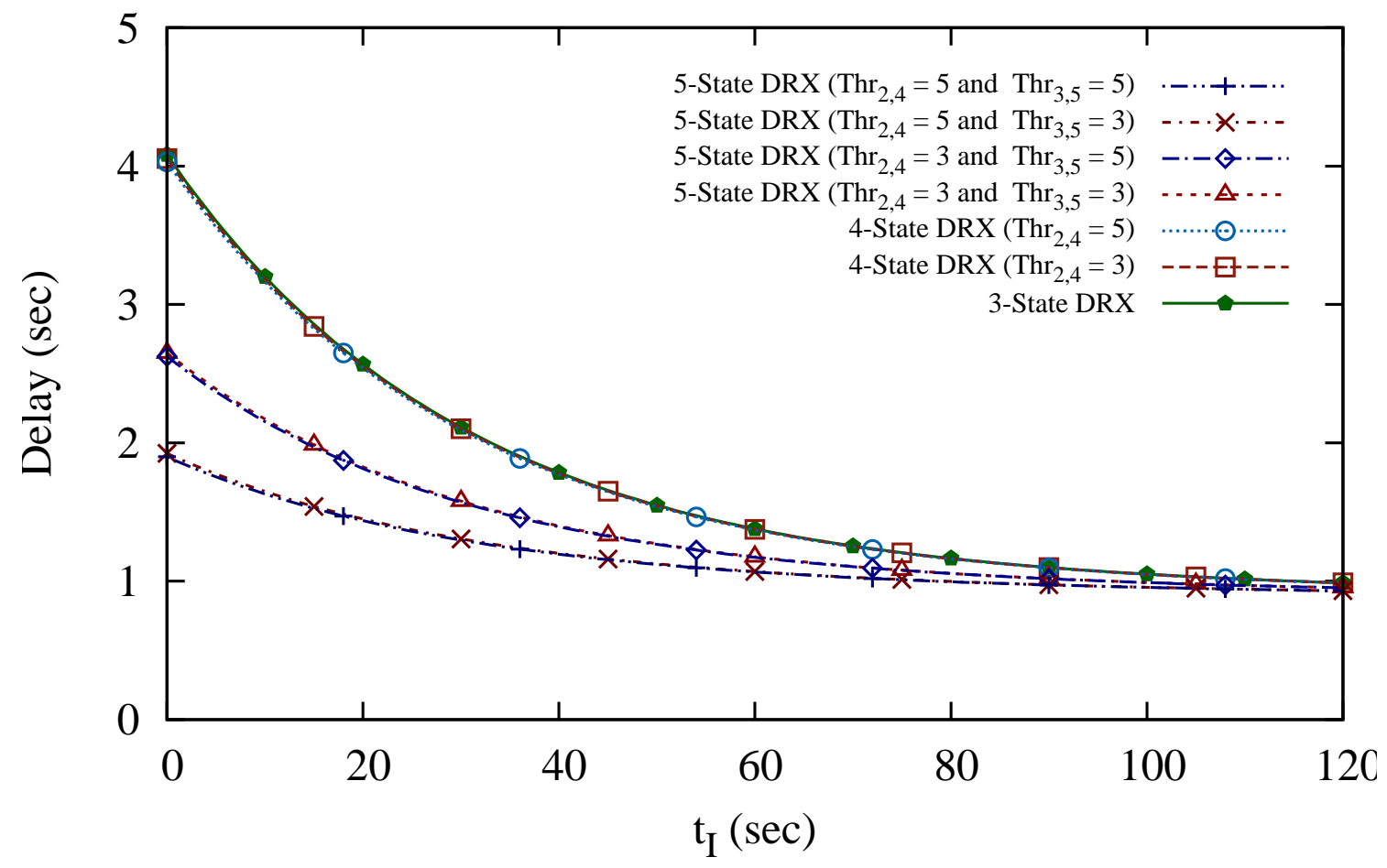

Figure 7: Performance evaluation 14 ith respect to Inactivity Timer $\left(t_{I}\right)$ 


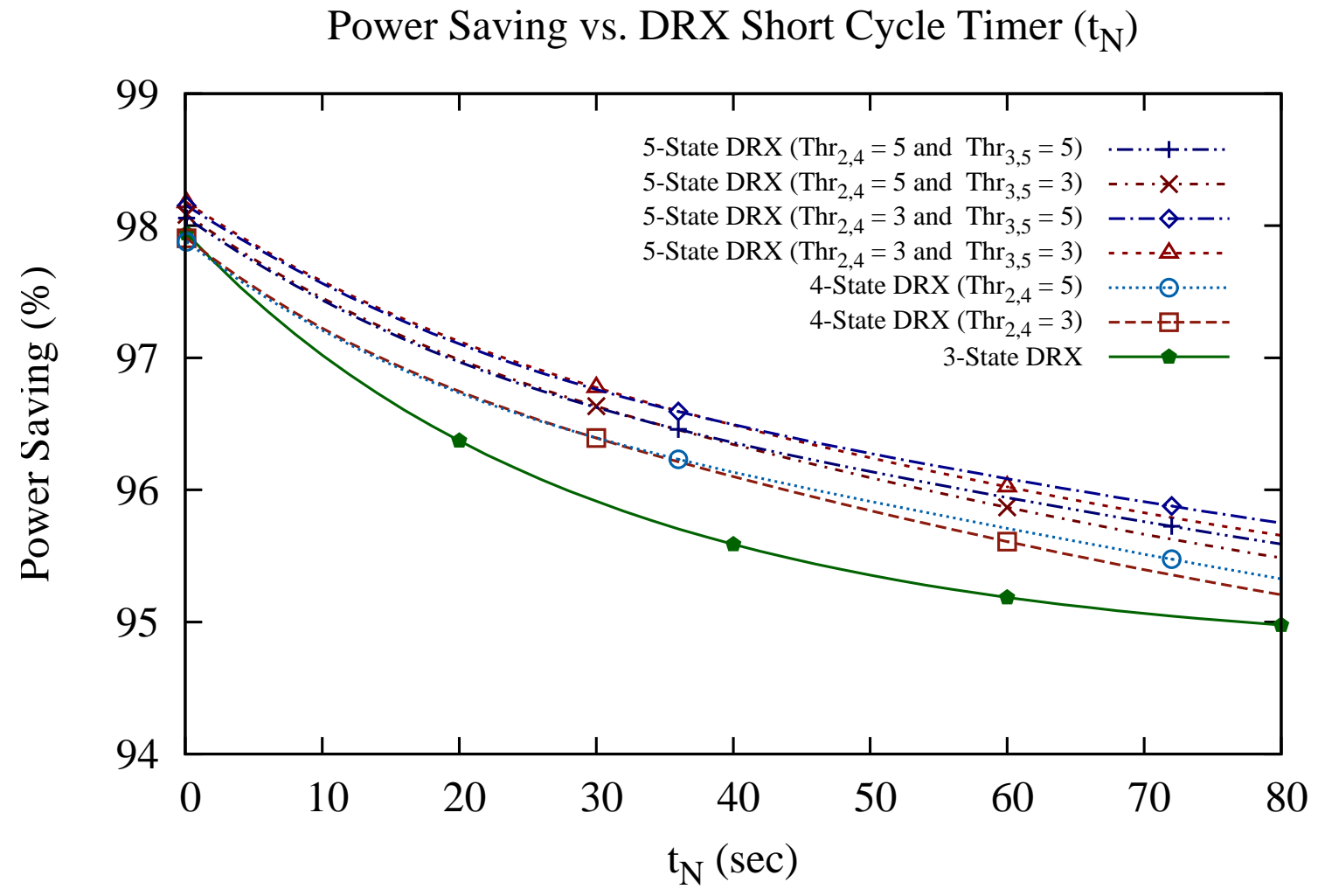

Delay vs. DRX Short Cycle Timer $\left(t_{N}\right)$

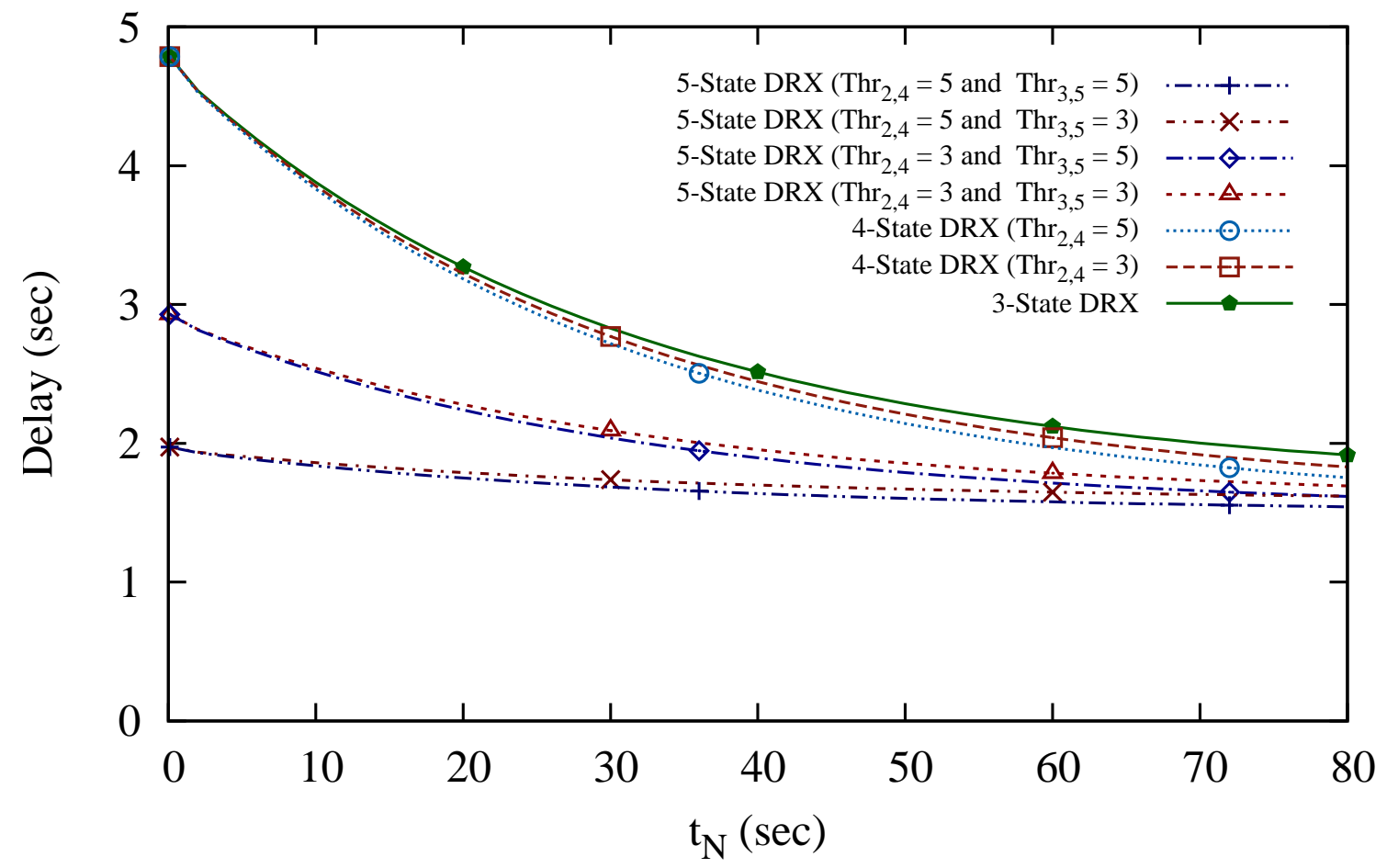

Figure 8: Performance evaluation with 15 respect to DRX Short Cycle Timer $\left(t_{N}\right)$ 

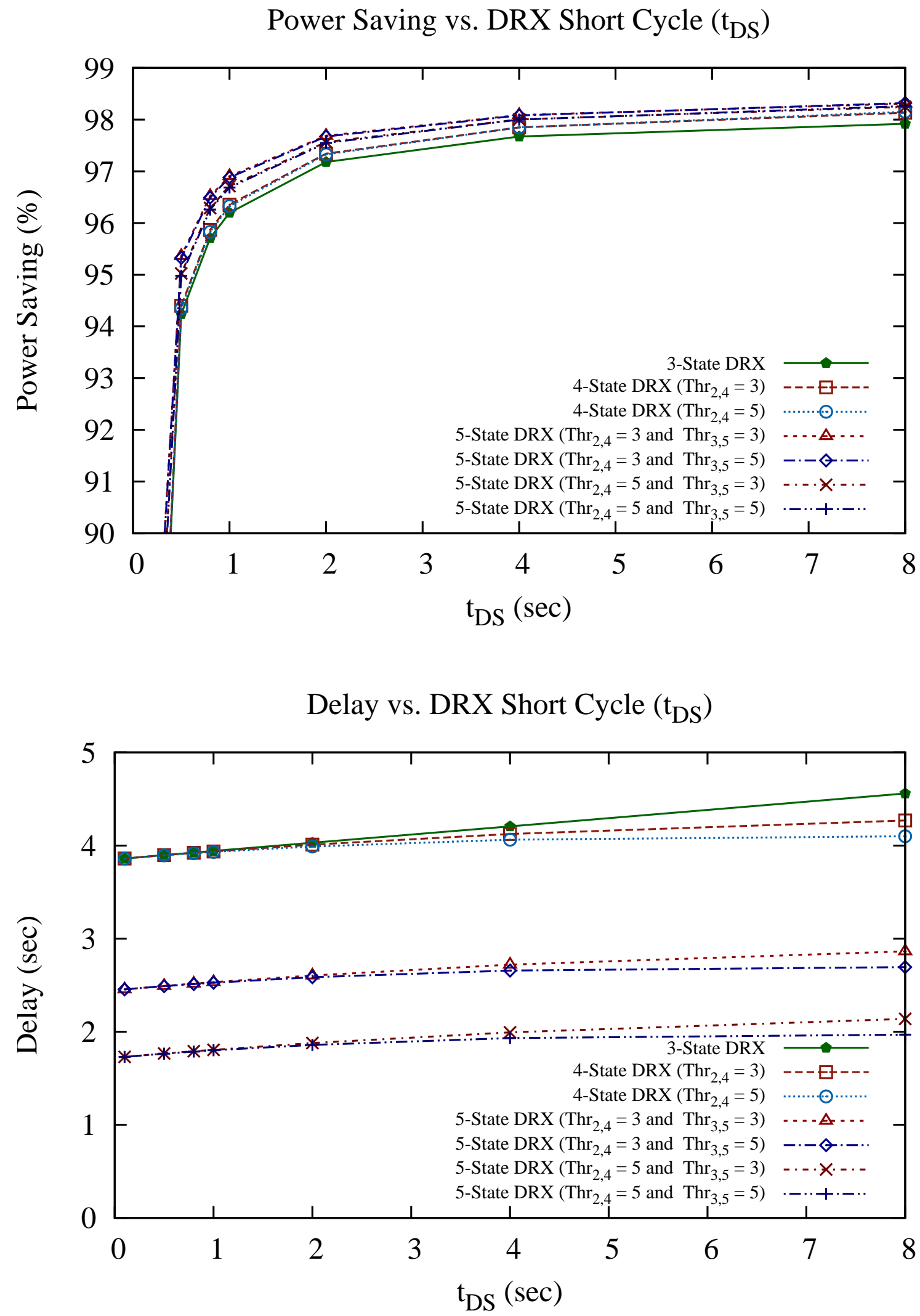

Figure 9: Performance evaluation wifh respect to DRX Short Cycle $\left(t_{D S}\right)$ 

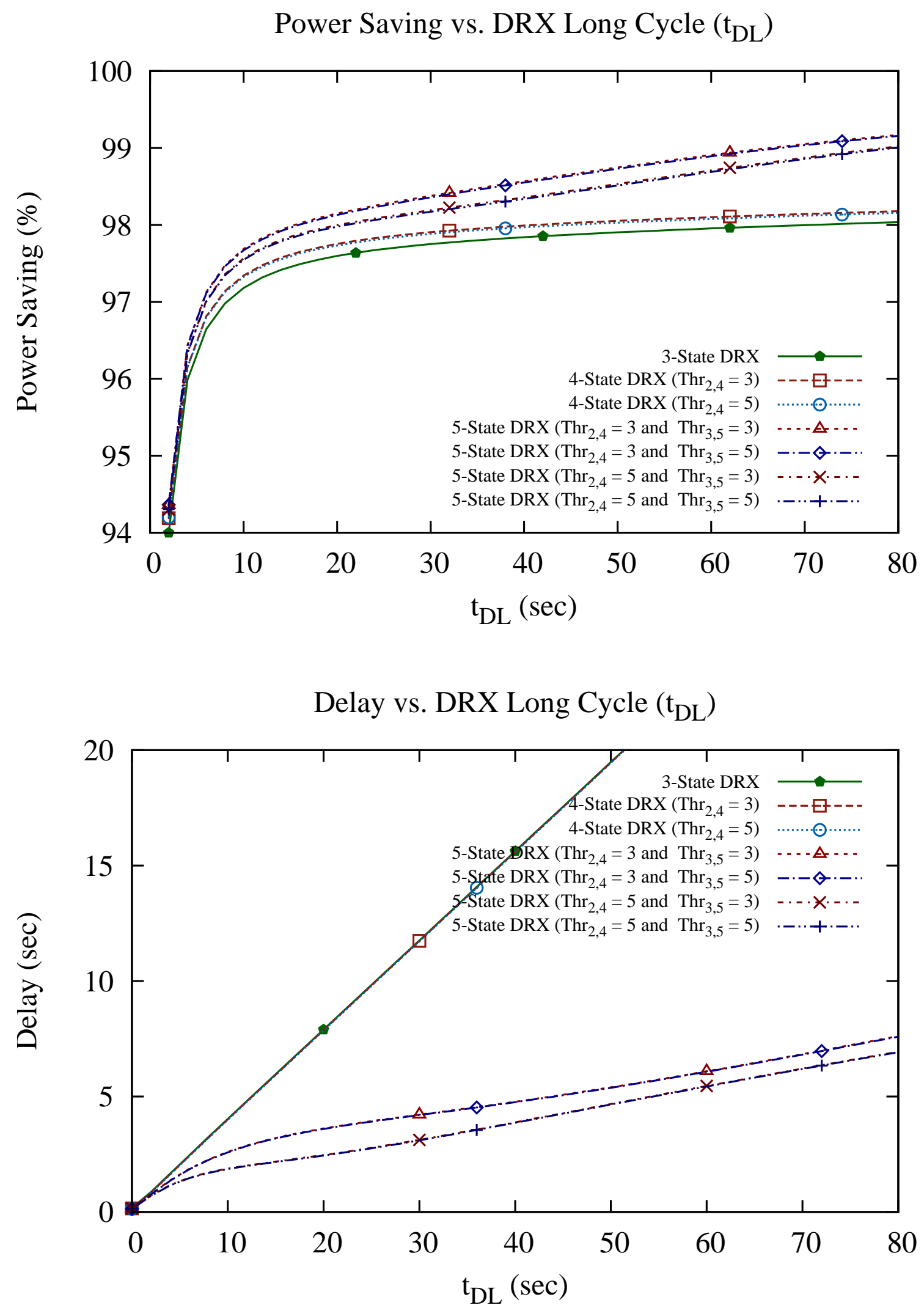

Figure 10: Performance evaluation with respect to DRX Long Cycle $\left(t_{D L}\right)$ 
The analytical result of the next timing parameter, shown in Figure 8, is regarding the DRX short cycle timer $\left(t_{N}\right)$. The results are similar to that of $t_{I}$, where the 4-state DRX has better performance than 3-state DRX and the 5 -state DRX has the best performance. From the outcome of our analysis, the approximate mean increments in power saving factor are $0.4 \%$ (4-state DRX) and $0.7 \%$ (5-state DRX) and for delay, the approximate mean decrements are $4 \%$ (4-state DRX) and 30\% (5-state DRX). These improvements in the extended DRX methods are instigated by the single-packet-active state which allows the UE during light traffic to stay in the sleep cycles thereby improving the power saving and also receive the incoming packets instantly to reduce the delay.

Now shifting the focus to the analytical results of the sleep cycles, i.e. the short DRX cycle and the long DRX cycle, where increasing the time period of the sleep cycles will increase both the power saving and delay. This is illustrated in Figures 9 and 10 where it is seen by increase in both the $t_{D S}$ and $t_{D L}$.

Figure 9 shows the comparison of performance of 4-state and 5-state against the 3 -state DRX with respect to $t_{D S}$. It was found that the power saving has improved slightly, around an average of $0.2 \%$ and $1.2 \%$ for the 4-state DRX and 5-state DRX respectively. On the other hand, the magnitude of the reduction in delay was much larger; an average of $1.7 \%$ and $45 \%$ decrements for the 4-state DRX and 5-state DRX, respectively.

Finally, the power saving and delay for $t_{D L}$ are shown in Figure 10. It is observed that the 5-state DRX mechanism has substantial improvement which is approximately $1 \%$ for power saving and $64 \%$ for delay. The difference between the 3-state and 4-state DRX is almost negligible because the single-packet-active state is only connected to deep sleep cycle in 5-state DRX only, as shown in Figure 6. For light traffic, the UE stays in the sleep cycle and does not transition to the active state. Hence, there is no delay during light traffic. For this reason, there is an improvement in delay for the 4-state and even greater improvement in the 5-state DRX. On the other hand, since the occurrence of light traffic is small the degree of improvements in power saving is also small.

\section{Conclusion}

In this paper, we presented a novel 4-state and 5-state 3GPP LTE DRX mechanisms. The proposed mechanisms were developed by augmenting (an) active state(s) to deep and/or light sleep cycle of standard 3-state DRX for handle a small burst of packets, thereby bypassing the process of returning to the timer-dependent active mode. We have generated analytical models using a semi-Markov process and evaluated these augmented DRX mechanisms against a standard 3-state DRX method for bursty packet data traffic.

In a standard 3-state DRX, an inbound packet forces an UE to start over the DRX process from the beginning, which means that the power saving factor mode kicks in only after inactivity timer in the active mode expires. This "waiting time" is a drawback of the timer-dependent 3-state DRX. The idea behind the proposed augmented DRX models is to reduce the "waiting time" by introducing (a) single-packet-active state(s). The single-packet-active site is not timer-dependent, and the state can be shifted directly from the light or deep sleep cycle. In other words, presence of a single-packet-active state allows a UE to bypass normal active state in case of handling a small burst of packets. This way, when there is an incoming packet during the sleep mode, the UE can go directly back to the power-saving sleeping mode immediately after receiving the packet (i.e. skipping the "waiting time" of the timer). With this transitory active site embedded in the sleep cycle, it is expected that reduced delay and improved power-saving would achieve. Overall, as anticipated, the analytical results from varying timing parameters showed that our augmented DRX (both 4-state and 5-state) improved power saving factor (ranging between $1 \%$ and $8 \%$ ) and reduced delay (ranging between $20 \%$ and 60\%) compared to the standard 3-state DRX (Figures 7-10). Furthermore, the magnitude of improvement for both delay and power-saving was somewhat greater in 5-state than in 4-state (Figures 7-10).In a standard 3-state DRX, an inbound packet forces an UE to start over the DRX process from the beginning, which means that the power saving factor mode kicks in only after inactivity timer in the active mode expires. This "waiting time" is a drawback of the timer-dependent 3-state DRX. The idea behind the proposed augmented DRX models is to reduce the "waiting time" by introducing (a) single-packet-active state(s). The single-packet-active site is not timer-dependent, and the state can be shifted directly from the light or deep sleep cycle. In other words, presence of a single-packet-active state allows a UE to bypass normal active state in case of handling a small burst of packets. This way, when there is an incoming packet during the sleep mode, the UE can go directly back to the power-saving sleeping mode immediately after receiving the packet (i.e. skipping the "waiting time" of the timer). With this transitory active site embedded in the sleep cycle, it is expected that reduced delay and improved power-saving would achieve. Overall, as anticipated, the analytical results from varying timing parameters showed that our augmented DRX (both 4-state and 5-state) 
improved power saving factor (ranging between $1 \%$ and $8 \%$ ) and reduced delay (ranging between 20\% and 60\%) compared to the standard 3-state DRX. Furthermore, the magnitude of improvement for both delay and power-saving was somewhat greater in 5-state than in 4-state. When adding extra state to the DRX model, however, the concomitant computational overhead should also be considered. It is expected that in 4- or 5-state, the gains will be greater than the losses since the energy and time required for this level of computation will be relatively low. Nonetheless, it may be interesting to see in the future, the trade-off analysis between the computational overhead and the multiple-state addition in the DRX technology.

The limitation of the current study (as well as other analytical DRX modeling studies) was that we made an assumption that the packet arrivals follow exponential distributions. However, as [21] points out, the packet arrivals do not follow any standard arrival process, particularly, when multiple applications are running in parallel in which resultant packet arrival process is a compound process whose parameters are unknown and have to be determined. Since the assumption is inevitable for any analytical modeling studies (even though the analytical results are normally reliable and relevant), it is ideal to validate the results using the gold standard, i.e. real traffic measurement.

The three major contributions of the current work are as follow. First, in terms of the study methodology, we applied semi-Markov process to model and analyze the DRX traffic behavior. We developed augmented DRX models (4-state and 5-state) extending from the standard 3-state DRX model by taking advantage of the flexibility of analytical modeling. For this, we applied semi-Markov process to model and analyze the DRX traffic behavior. Second, we presented the effectiveness of the augmented DRX mechanisms with the single-packet-active state (or stationary active site) embedded in the sleep cycle. With the high bandwidth and high data rate enabled by LTE, subscribers are keen to have newer (and more) applications which tend to be power hungry and delay sensitive. This is the reason why LTE should have a DRX mechanism with higher effectivity. The proposed technique is one way to maximize LTEs capacity to solve battery-life problem and delay problem for the end users UE. Finally, our overall contribution is that we demonstrated an innovative development of technique, i.e. the addition of states to the generic model, significantly improved the delay and energy efficiency without sacrificing each other. Application of simple and effective methodologies, such as the ones proposed in this paper, may benefit the development of soon coming $5 \mathrm{G}$ networks.

\section{Acknowledgment}

Scott Fowler was partially supported by the EC-FP7 Marie Curie CIG grant, Proposal number: 294182.

\section{References}

[1] 3GPP TS 36.300, Evolved Universal Terrestrial Radio Access (E-UTRA) and Evolved Universal Terrestrial Radio Access (E-UTRAN); Overall description; Stage 2, TS 36.300, 3rd Generation Partnership Project (3GPP) (Sep. 2008)

URL http://www. 3gpp.org/ftp/Specs/html-info/36300.htm

[2] C. Bontu, E. Illidge, Drx mechanism for power saving in lte, IEEE Communications Magazine 47 (6) (2009) $48-55$.

[3] E. Hwang, K. J. Kim, J. J. Son, B. D. Choi, The power-saving mechanism with periodic traffic indications in the ieee $802.16 \mathrm{e} / \mathrm{m}$, IEEE Transactions on Vehicular Technology 59 (1) (2010) 319-334.

[4] S. Jin, K. Han, S. Choi, Idle mode for deep power save in ieee 802.11 wlans, Journal of Communications and Networks 12 (5) (2010) 480-491.

[5] F.-W. Li, Y.-Q. Zhang, L.-W. Li, Enhanced discontinuous reception mechanism for power saving in td-lte, in: IEEE International Conference on Computer Science and Information Technology (ICCSIT), Vol. 9, 2010, pp. 682-686.

[6] Y. Mihov, K. Kassev, B. Tsankov, Analysis and performance evaluation of the drx mechanism for power saving in lte, in: IEEE 26th Convention of Electrical and Electronics Engineers in Israel (IEEEI), 2010, pp. 520-0524.

[7] S.-R. Yang, P. Lin, P.-T. Huang, Modeling power saving for gan and umts interworking, IEEE Transactions on Wireless Communications 7 (12) (2008) 5326-5335.

[8] S.-R. Yang, Y.-B. Lin, Modeling umts discontinuous reception mechanism, IEEE Transactions on Wireless Communications 4 (1) (2005) 312-319.

[9] S.-R. Yang, S.-Y. Yan, H.-N. Hung, Modeling umts power saving with bursty packet data traffic, IEEE Transactions on Mobile Computing 6 (12) (2007) 1398-1409.

[10] S. Yang, M. Yoo, Y. Shin, Adaptive discontinuous reception mechanism for power saving in umts, IEEE Communications Letters 11 (1) (2007) 40-42.

[11] J.-H. Yeh, J.-C. Chen, C.-C. Lee, Comparative analysis of energy-saving techniques in 3gpp and 3gpp2 systems, IEEE Transactions on Vehicular Technology 58 (1) (2009) 432-448. 
[12] L. Zhou, H. Xu, H. Tian, Y. Gao, L. Du, L. Chen, Performance analysis of power saving mechanism with adjustable drx cycles in 3gpp lte, in: IEEE Vehicular Technology Conference (VTC-Fall), 2008, pp. 1-5.

[13] J. Wigard, T. Kolding, L. Dalsgaard, C. Coletti, On the user performance of lte ue power savings schemes with discontinuous reception in lte, in: IEEE International Conference on Communications Workshops, 2009, pp. 1-5.

[14] M. Polignano, D. Vinella, D. Laselva, J. Wigard, T. Sorensens, Power savings and qos impact for voip application with drx/dtx feature in lte, in: IEEE Vehicular Technology Conference (VTC-Spring), 2011, pp. 1-5.

[15] S. Jha, A. Ko, R. Vannithamby, Optimization of discontinuous reception (drx) for mobile internet applications over lte, in: IEEE Vehicular Technology Conference (VTC-Fall), 2012, pp. 1-5.

[16] S. Jin, D. Qiao, Numerical analysis of the power saving in 3gpp lte advanced wireless networks, IEEE Transactions on Vehicular Technology 61 (4) (2012) 1779-1785.

[17] 3GPP TS 25.304, User Equipment (UE) procedures in idle mode and procedures for cell reselection in connected mode, TS 25.304, 3rd Generation Partnership Project (3GPP) (Sep. 2005).

URL http://www.3gpp.org/ftp/Specs/html-info/25304.htm

[18] 3GPP TS 36.321, Evolved Universal Terrestrial Radio Access (E-UTRA); Medium Access Control (MAC) protocol specification, TS 36.321, 3rd Generation Partnership Project (3GPP) (Sep. 2008).

URL http://www.3gpp.org/ftp/Specs/html-info/36321.htm

[19] E. Liu, J. Zhang, W. Ren, Adaptive drx scheme for beyond 3g mobile handsets, in: IEEE Global Telecommunications Conference (GLOBECOM), 2011, pp. 1-5.

[20] ETSI, Universal Mobile Telecommunications System (UMTS); Selection procedures for the choice of radio transmission technologies of the UMTS, TR 101 112, European Telecommunications Standards Institute (ETSI) (Apr. 1998).

URL http://www.etsi.org/deliver/etsi_tr/101100_101199/101112/03.02.00_60/ tr_101112v030200p.pdf

[21] R. Karthik, A. Chakrapani, Practical algorithm for power efficient drx configuration in next generation mobiles, in: IEEE INFOCOM, 2013, pp. 1106-1114. 